\title{
A Rectangular-shaped Time-delay Line Inspired by CRLH TL Unit Cell for UWB Operation
}

\author{
J. Zhang, S.W. Cheung and T.I. Yuk \\ Department of Electrical and Electronic Engineering \\ The University of Hong Kong \\ Hong Kong, China \\ zhangjun@eee.hku.hk, swcheung@eee.hku.hk, tiyuk@eee.hku.hk
}

\begin{abstract}
This paper presents the design of a rectangular-shaped ultra-wideband (UWB) time-delay line inspired by the use of composite right/left-handed transmission line (CRLH TL) unit cells. A rotated version of a conventional CRLH TL unit cell is used as the basic elements to achieve UWB operation. For comparison, time-delay lines using the right-handed transmission line (RH TL) and CRLH TL unit cells are also studied, fabricated and tested. Simulation and measurement results show that our proposed time-delay lines have high return loss, low insertion loss, UWB operation and much longer time delays than that of the time-delay line based on RH TL.
\end{abstract}

Keywords-compact; rectangular-shaped time-delay line; UWB; CRLH TL

\section{INTRODUCTION}

Microwave time-delay lines find many applications in different areas such as radar and phased array systems and other telecommunication systems. In the design of microwave time-delay lines, the size, return loss and insertion loss are all important design parameters to be concerned.

Metamaterials, also known as left-handed materials (LHMs), have negative permittivity and permeability which are not commonly found in nature. Its concept was first investigated by Veselago in 1968 [1]. Although the properties of LHMs promised for a large diversity of novel applications and devices, LHMs did not attract much attention until it was found that the materials could be realized using a general transmission line (TL) approach [2]. Practical LH TLs also have the right-handed $(\mathrm{RH})$ effects, so LHMs realized using TLs are called composite right/left handed transmission lines (CRLH TLs). CRLH TL can be used to design many different microwave components such as phase shifters, antennas and bandpass filters, etc. Recently, time-delay lines based on CRLH TL have been proposed and studied [3-6]. However, these designs have relatively narrow bandwidths.

In this paper, we study using CRLH TL unit cell [3] as the basic elements to design time-delay lines and the rotated version of the unit cell to design time-delay lines for ultra-wide band (UWB) applications. Long and narrow time-delay lines can not be easily fitted to practical circuits which normally have relatively even widths and lengths. In this paper, we propose to bend the wideband time-delay line to form a rectangular shape so that it can be placed on a rectangular area of the PCB or used to enclose large circuits on a PCB for easy fitting to practical circuits. Simulation results using CST MWS 2009 and measurement results show that our proposed timedelay lines have UWB operation bandwidths from $0.1-10 \mathrm{GHz}$, high return loss of more than $15 \mathrm{~dB}$ and low insertion loss of less than $1 \mathrm{~dB}$.

\section{TIME-DELAY LINE USING TRANSMISSION LINE}

For a TL with a length of $L$, the time for a signal to propagate through is given by:

$$
t_{\text {delay }}=\frac{L}{\lambda f}=\frac{L \times \beta}{2 \pi f}
$$

where $f$ and $\beta$ are the operating frequency and phase constant, respectively. Thus if a TL is used as a time-delay line, both the length of the transmission line and the phase constant $\beta$ can be used to adjust the amount of time delay for signals to propagate through. For a fixed time delay, a larger $\beta$ implies a shorter transmission line is required. Traditional TL, also known as RH $\mathrm{TL}$, has a small phase constant $\beta$ and so a very long length is needed to produce a large time delay, which leads to a large insertion loss.

\section{MODEL OF CRLH TL UNIT CELL}

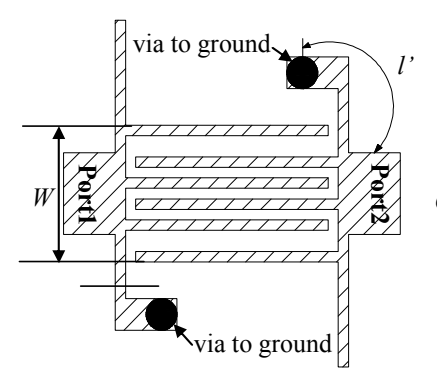

(a)

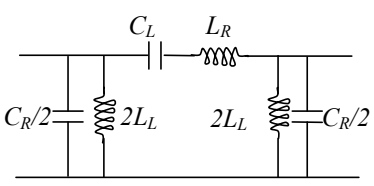

(b)
Figure 1 (a) Structure and (b) equivalent circuit of conventional CRLH TL unit cell

The general schematic diagram and the equivalent circuit of a CRLH TL unit cell [3] with six fingers are shown in Fig. 1. In 
Fig. 1(b), the series inductance $L_{R}$ and capacitance $C_{L}$ are the inductance along the fingers and the coupling capacitance between the fingers, respectively, the shunt inductance $L_{L}$ is realized by the two fingers each having a via at the ends to the ground, and the shunt capacitance $C_{R}$ is the stray capacitance of the fingers. The expressions for $L_{R}, C_{R}, L_{L}$ and $C_{L}$ are, respectively, given by $[7,8]$ :

$$
\begin{gathered}
L_{R}=\frac{Z_{0} \sqrt{\varepsilon_{r e}}}{c} l \\
C_{R}=\frac{\sqrt{\varepsilon_{r e}}}{Z_{0} c} l \\
C_{L}=\left(\varepsilon_{r}+1\right) l\left\{4.409(N-3) \tanh \left[0.55\left(\frac{h}{W}\right)^{0.45}\right]\right. \\
\left.\left.\left.+9.92 \tanh \left[0.52\left(\frac{h}{W}\right)^{0.5}\right]\right\} \times 10^{-12}\right]\right\} \\
L_{L}=\frac{\mu_{0}\left[h \cdot \ln \left(\frac{h+\sqrt{r^{2}+h^{2}}}{2 \pi}\right)+\frac{3}{2}\left(r-\sqrt{r^{2}+h^{2}}\right)\right]}{+\frac{Z_{0} \sqrt{\varepsilon_{r e}}}{c} l^{\prime}}
\end{gathered}
$$

where $h$ is the thickness of the substrate, $r$ is the radius of the ground via, $\varepsilon_{r}$ is the relative dielectric constant, $\varepsilon_{r e}$ is the effective dielectric constant, $l$ ' is the distance from the ground via to the port, $l$ is the length of the finger, $N$ is the number of the fingers, $W$ is the total width of all the fingers together and $Z_{0}$ is the characteristic impedance of each of the fingers. The phase constant $\beta$ of such CRLH TL unit cell is [9]:

$$
\beta=\omega \sqrt{L_{R} C_{R}}-\frac{1}{\omega \sqrt{L_{L} C_{L}}}
$$

(2) - (6) show that the structural parameters $L_{R}, C_{R}, L_{L}$ and $C_{L}$ determine $\beta$ and hence can be used to adjust the time delay of the unit cell given in (1).

\section{DESIGN OF TIME-DELAY LINES INSPIRED BY CRLH TL UNIT CELL}

\section{A. Narrowband time-delay line}

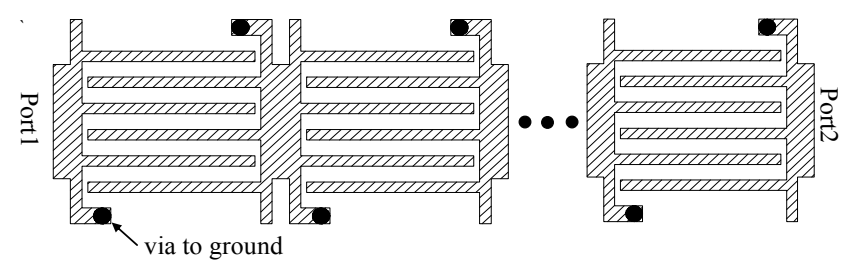

Figure 2 Narrowband time-delay line by cascading conventional CRLH TL unit cells
Since the structural parameters $L_{R}, C_{R}, L_{L}$ and $C_{L}$ of the CRLH TL unit cell can be used to determine the value of $\beta$, we can design a unit cell to maximize the time delay through (1) and use it as the basic elements to design time-delay lines, i.e., cascading a number of the unit cells together, as shown in Fig. 2 , to produce a long delay time. However, computer simulation results have shown such time-delay lines have narrow bandwidths.

\section{B. Wideband time-delay line}

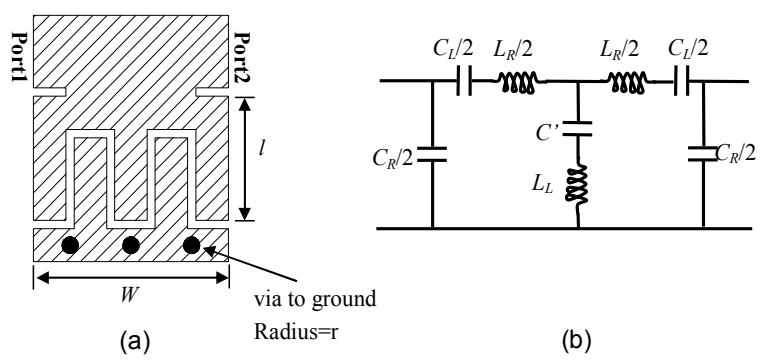

Figure 3 Rotated structure and (b) equivalent circuit of the unit cell

To increase the operation bandwidth of the time-delay line, we propose to rotate the CRLH TL cell of Fig. 1(a) by $90-$ degree, as shown in Fig. 3(a), with the equivalent circuit shown in Fig. 3(b). Simulation studies have shown that such unit cell has a much wider bandwidth. When this unit cell is used as the basic periodic elements to design time-delay lines with long time delays, we also propose to reduce the number of fingers to five (an odd number) to make the unit cell a symmetrical structure, so that the two S-parameters, $S_{11}$ and $S_{21}$, of the unit cell are equal. Then when identical unit cells are used in cascade to provide a long time delay, there is no need to perform any matching between the unit cells. The time-delay lines constructed by cascading 16 conventional CRLH TL unit cells and 16 wideband unit cells are shown in Figs. 4(b) and $4(c)$, respectively.

\section{Rectangular-shaped time-delay line}

Practical circuits normally occupy areas with relatively even widths and lengths on the PCBs which may not have the space to accommodate a long and narrow time-delay line. Here we propose to bend the wideband time-delay line into a rectangular shape, so that it occupies a rectangular area on a PCB. For a long time-delay line, circuits may also be fabricated inside the rectangular time-delay line. A rectangular-shaped time-delay line using 16 wideband unit cells is shown in Fig. 4(d).

\section{RESULTS AND DiSCUSSIONS}

For the purpose of comparison, we have designed timedelay lines using RH TL, 16 conventional CRLH TL unit cells, 16 wideband unit cells, and 16 wideband unit cells in a rectangular shape, with the layouts shown in Fig. 4. They all have a length of about $60 \mathrm{~mm}$. The designs have been optimized using the EM simulation tool CST MWS 2009. In the optimization process, the width of each finger was set to 0.2 $\mathrm{mm}$ which is the smallest dimension we could make in our lab. Results have shown that the length of the fingers in the rotated 
unit cells has significant effects on the return loss, so we mainly optimized the length of the fingers. The dimensions of the unit cells were optimized based on the criteria of having the return loss of more than $15 \mathrm{~dB}$ and the largest phase constant $\beta$. The final optimization results of these designs were used for fabrication of the time-delay lines on a F4B-2 PCB with a thickness of $0.8 \mathrm{~mm}$ and permittivity of 2.65 , as shown in Fig. 5. The return loss, insertion loss and time delay of all these time-delay lines have been simulated and measured.

Figure 6 shows the simulated and measured return loss and insertion loss of the time-delay lines using the RH TL, the conventional CRLH TL unit cells and the wideband unit cells. The time delays of these time-delay lines are shown in Fig. 7. It can be seen that the simulated and measured results show good agreements. The time-delay line using the RH TL has quite a high return loss as can be seen in Fig. 6(a) and a low insertion loss of less than $1 \mathrm{~dB}$, but the time delay achieved is only 300 ps. The narrowband time-delay line achieves a slightly longer time delay of $1800 \mathrm{ps}$, about $60 \mathrm{ps}(3.5 \%)$ longer than that of the wideband time-delay line, but it has a narrower bandwidth of only 4-6 GHz and a larger insertion loss of about $2 \mathrm{~dB}$ within the 4-6 GHz pass band as shown in Fig. 6(b).

The return loss and insertion loss for the wideband timedelay line are shown in Fig. 6(c). The measured return loss is more than $15 \mathrm{~dB}$ and the insertion loss is less than $1 \mathrm{~dB}$ across the whole frequency band from $0.1-10 \mathrm{GHz}$. Figure 7 shows that it has a time delay of $1740 \mathrm{ps}$ across the whole frequency band tested, nearly 6 times longer than that of the RH TL.

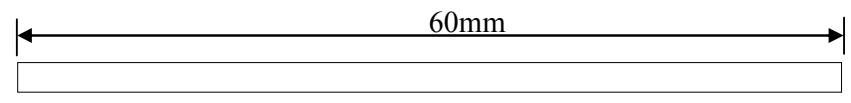

(a)

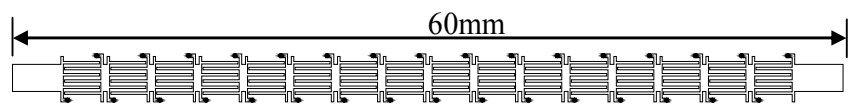

(b)

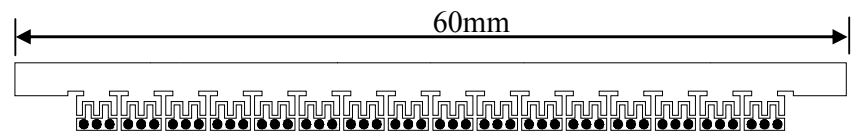

(c)

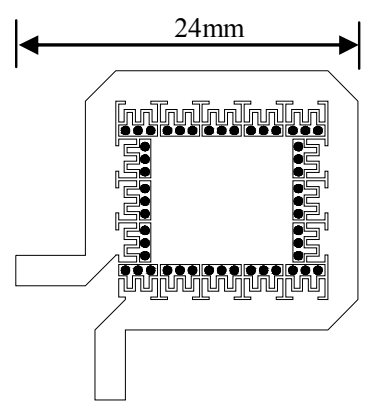

(d)

Figure 4 Layouts of time-delay lines using

(a) RH TL, (b) conventional CRLH TL unit cells, (c) wideband unit cells and (d) wideband unit cells in rectangular shape

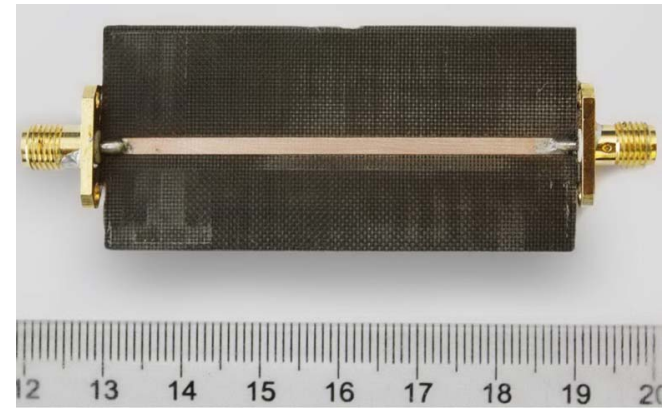

(a)

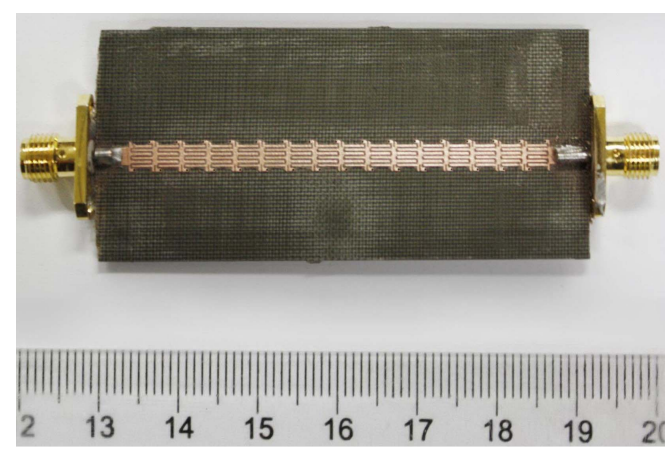

(b)

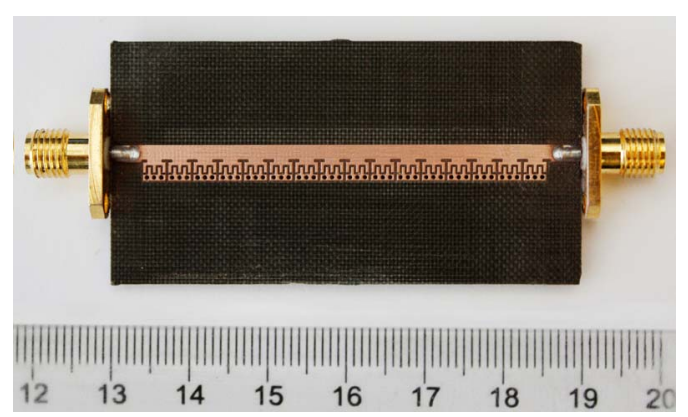

(c)

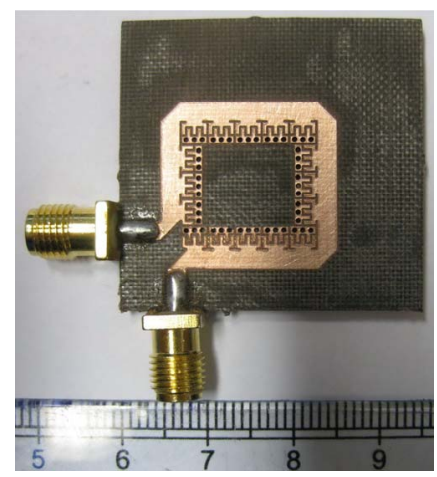

(d)

Figure 5 Prototyped time-delay lines using

(a) RH TL, (b) conventional CRLH TL unit cells, (c) wideband unit cells and (d) wideband unit cells in rectangular shape 


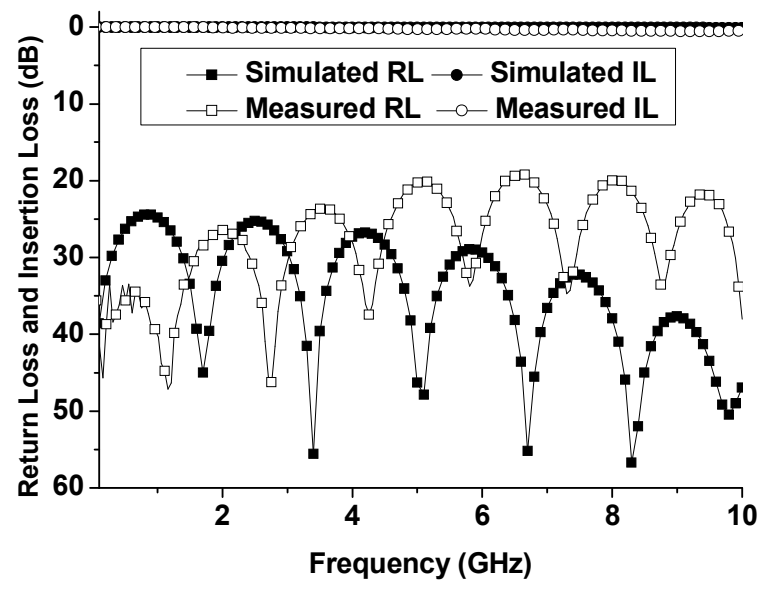

(a)

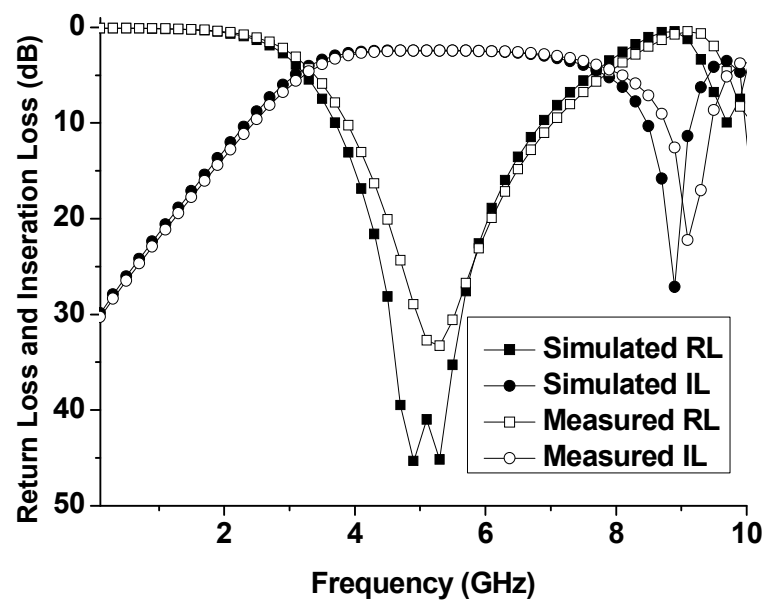

(b)

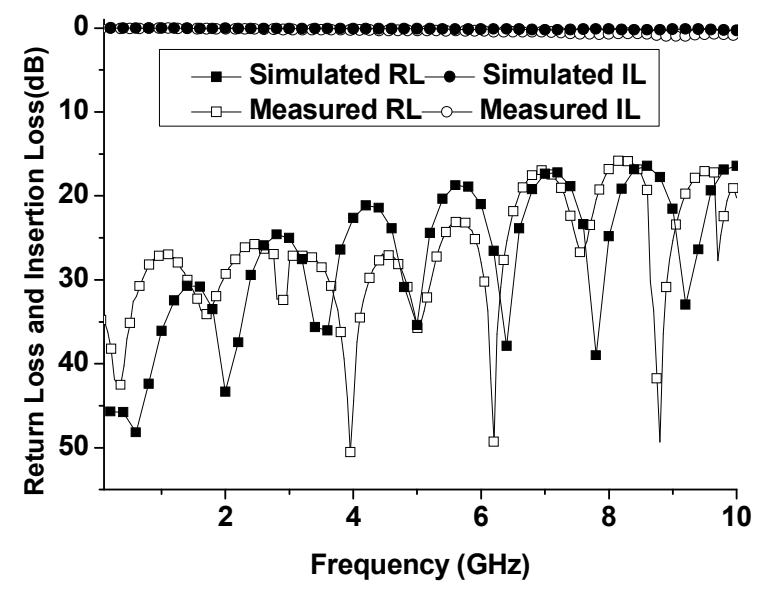

(c)

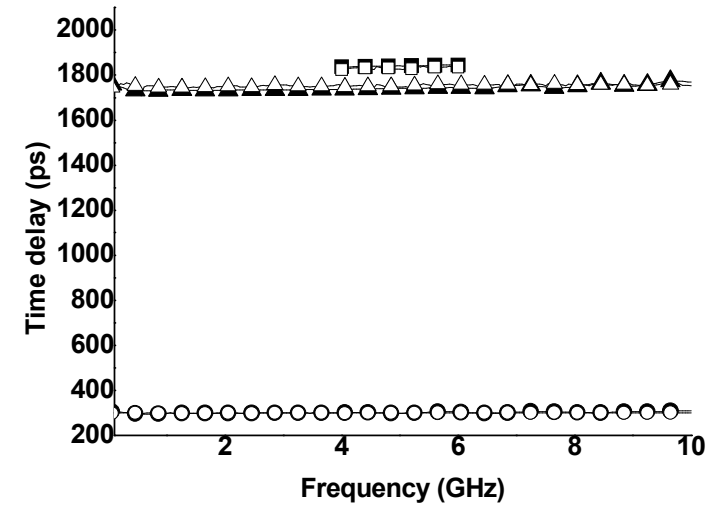

- RH TL(simulated)

-O- RH TL(measured)

- conventional CRLH TL unit cells(simulated)

$\rightarrow-$ conventional CRLH TL unit cells(measured)

$\triangle$ rotated wideband unit cells(simulated)

$\triangle-$ rotated wideband unit cells (measured)

Figure 7 Simulated and measured time delays of different time-delay lines

For the rectangular-shaped wideband time-delay line of Fig. 4(d), the simulated and measured return loss and insertion loss are shown in Figs. 8 and 9, respectively. Again, the simulated and measured results show good agreements. Figure 8 shows that the measured return loss is more than $15 \mathrm{~dB}$ and the insertion loss is less than $1 \mathrm{~dB}$ across the whole tested frequency band. The time delay is about 1780 ps across the whole frequency band of $0.1-10 \mathrm{GHz}$, about 6 times longer than that of the time-delay line for the RH TL. The time delay is slightly longer than that of the wideband time-delay line. This is because the bending slightly increases the overall length. The results show that the rectangular shape does not change the properties much, but makes the fitting to practical circuits on the PCBs much easier.

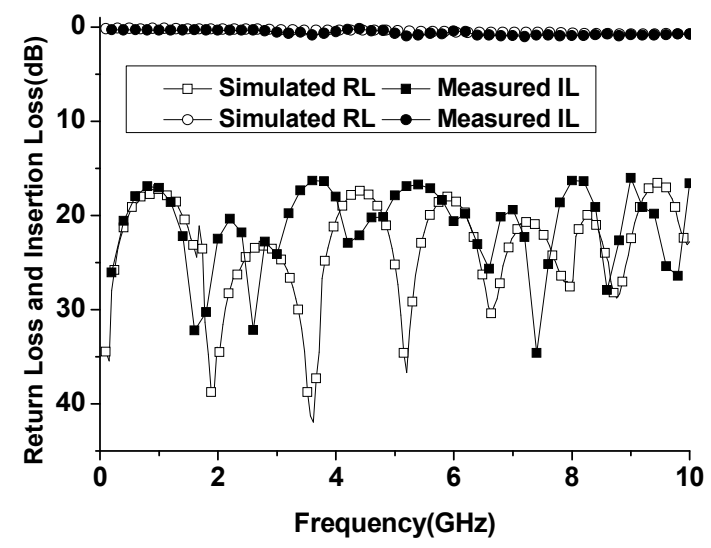

Figure 8 Simulated and measured return loss and insertion loss of time-delay line using rotated wideband unit cells in rectangular shape
Figure 6 Simulated and measured return loss and insertion loss of time-delay lines using (a) RH TL, (b) conventional CRLH TL unit cells and (c) wideband unit cells 


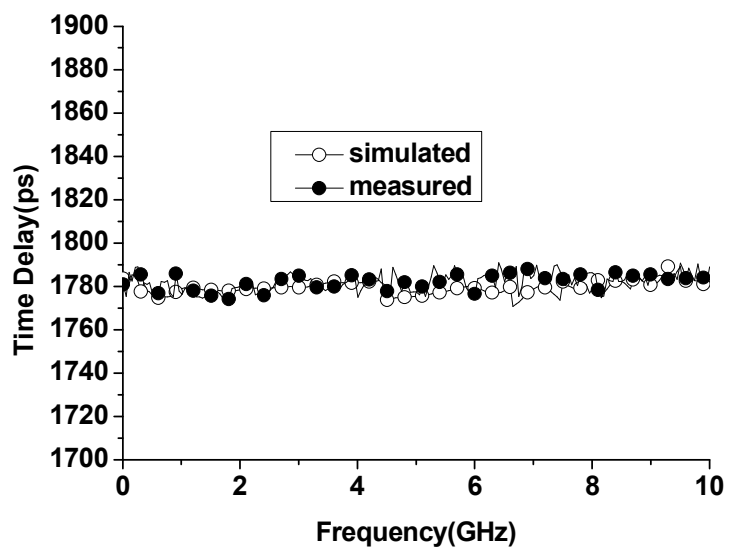

Figure 9 Simulated and measured time delay of time-delay line using rotated wideband unit cells in rectangular shape

\section{CONCLUSIONS}

A rectangular-shaped UWB time-delay line constructed by cascading a number of rotated versions of the conventional CRLH TL unit cells has been proposed, studied and fabricated. Three other time-delay lines using the conventional CRLH TL unit cells, wideband unit cells and RH TL have also been designed, optimized and fabricated for comparison. Simulation and measurement results have demonstrated that, for the length of $60 \mathrm{~mm}$, our proposed rectangular-shaped wideband time-delay line has a much longer time delay of 1780 ps, about 6 times longer than that of using the RH TL, yet having a much wider operating bandwidth of $0.1-10 \mathrm{GHz}$, a high return loss and a low insertion loss. The time-delay line has a rectangular shape and so can be easily integrated with practical circuits on a PCB.

\section{REFERENCES}

[1] V. Veselago, "The electrodynamics of substances with simultaneously negative values of $\varepsilon$ and $\mu$," Soviet Physics Uspekhi, vol. 10, pp. 509514, 1968.

[2] C. Caloz, H. Okabe, T. Iwai, and T. Itoh, "Transmission line approach of left-handed (LH) materials," Proc. USNC/URSI National Radio Science Meeting, vol. 1, pp. 39, San Antonio, Texas, June 2002,

[3] J. Zhang, Q. Zhu, Q. Jiang and S.J. Xu, "Design of time delay lines with periodic microstrip line and composite right/left-handed transmission line," Microwave and Optical Technology Letters, vol. 51, pp. 16791682, 2009.

[4] S. Pasakawee and Z. Hu, "Left-handed microstrip delay line implemented by Complementary Split Ring Resonators (CSRRs)," in Asia Pacific Microwave Conference 2009, pp. 599-601, Singapore, 2009.

[5] Choul-Young Kim, Jaemo Yang, Dong-Wook Kim and Songcheol Hong, "A K-Band CMOS Voltage Controlled Delay Line Based on an Artificial Left-Handed Transmission Line," IEEE Microwave and Wireless Components Letters, vol. 18, pp. 731-733, 2008.

[6] Wenjia Tang and Hongjoon Kim, "Compact, tunable large group delay line," in Wireless and Microwave Technology Conference 2009, Clearwater, FL, 2009.

[7] I. Bahl, Lumped elements for RF and microwave circuits, Artech House: Boston \& London, 2001

[8] E. G. Marc and A. P. Robert, "Modeling via hole grounds in Microstrip," IEEE Microwave and Guided Wave Letters, vol. 1, pp. 135137, 1991.

[9] A. Lai, T. Itoh and C. Caloz, "Composite Right/Left-Handed Transmission Line Metamaterials," IEEE Microwave Magazine, vol. 5, pp. 34-50, 2004. 